\title{
MR diffusion tensor imaging of the spinal cord: can it help in early detection of cervical spondylotic myelopathy and assessment of its severity?
}

\author{
Talaat Ahmed Abd El Hameed Hassan ${ }^{1 *}$ (D), Ramy Edward Assad ${ }^{2}$ and Shaimaa Atef Belal ${ }^{2}$
}

\begin{abstract}
Background: The aim of this study is to evaluate the potential application of MR diffusion tensor imaging (with calculation of fractional anisotropy (FA) values) in assessment of the spondylotic cervical spinal canal compromise and comparison with the information issued from conventional MR sequences for early detection of cervical spondylotic myelopathy (CSM). Thirty patients (11 males and 19 females) were included in this study; age ranged from 22 to 70 years (mean age $=44$ ). All patients had conventional and diffusion tensor imaging (DTI) examinations of the cervical spine for detection and assessment of degree of cervical cord myelopathy. FA values of the whole cord circumference and at 3, 6, 9, 12 o'clock positions of the normal cord (opposite to C2), opposite to the most affected disc, and below the level of the most affected disc were measured.

Results: High statistically significant $P$ values were obtained when comparing the FA values of the normal cord with the cord opposite to the most affected disc, the normal cord with the cord below the affected disc and the cord at the level of the most affected disc with the cord below the level of the most affected disc.

Conclusions: DTI of the cervical spinal cord with FA measurement in patients with cervical spondylosis helps in early detection of cervical cord compressive myelopathy prior to appearance of changes in conventional MRI, which can improve the clinical outcome and help in treatment plans.
\end{abstract}

Keywords: Diffusion tensor imaging, Anisotropy, Spondylosis, Spinal cord compression

\section{Background}

Conventional MR examination showed a significant role in the diagnosis of cervical spondylosis, which is a common degenerative disease of the spine in the elderly and manifested by protrusion of the intervertebral discs, osteophytosis formation, narrowing of the cervical canal, etc. [1].

Cervical spondylosis can cause compressive myelopathy of the cervical spinal cord and motor dysfunction. The treatment of this problem is usually operative intervention. Early MRI diagnosis of this problem and early therapeutic intervention is associated with good prognosis and better clinical outcome [2].

\footnotetext{
* Correspondence: talaathassan38@yahoo.com

${ }^{1}$ Radiology Department, Faculty of Medicine, Cairo University, Giza, Egypt

Full list of author information is available at the end of the article
}

Evaluation of the morphological changes in cervical spondylosis is usually done by using conventional MRI, but there is sometimes discrepancy between clinical symptoms and MR imaging findings with weak correlation [3].

The compressive cord myelopathy opposite to a certain level in the spinal canal in cases of cervical spondylosis is manifested by high signal inside the cord in T2WI. In patients with chronic symptoms of cervical spondylosis, this T2WI sign appears late and shows low sensitivity for the detection of spinal cord myelopathies [2].

Diffusion tensor imaging (DTI) shows better sensitivity when compared to the conventional T2WI sequence in the early detection of cervical spondylotic myelopathy (CSM) because it shows abnormalities in the spinal cord 
Table 1 Distribution of cases according to clinical presentation

\begin{tabular}{ll}
\hline Clinical presentation & Number of cases \\
\hline Neck pain & $12 / 30(40 \%)$ \\
Brachialgia & $2 / 30(6.7 \%)$ \\
Both & $16 / 30(53.3 \%)$
\end{tabular}

before the development of $\mathrm{T} 2$ hyperintensity in patients with CSM [4].

Conventional MRI is less sensitive when compared to DTI in detection of subtle pathological changes of the spinal cord [5].

Among the different DTI parameters, the fractional anisotropy (FA) parameter shows higher sensitivity and specificity for early detection of spinal cord subtle abnormalities when compared with conventional T2WI [2].

The abnormalities of different DTI parameters could show focal or extensive myelopathic changes in CSM because spinal tracts are not affected by the same degree in the myelopathic cervical cord [6].

\section{Methods}

This prospective study included thirty patients and was conducted in the period from July 2015 to October 2017, following approval by our institutional review board. Patients were referred from the neurosurgery outpatient clinics to the radiology department. Patients gave informed consent prior to the MR scanning. Each patient included in the study was subjected to full history taking. The most frequent neurological clinical presentations given by the patients were neck pain and brachialgia.

\section{Inclusion criteria}

Patients with neck pain that is referred to the arms, weakness or numbness in the arms, and/or difficulty in performing activities involving fine motor skills (handwriting, buttoning a shirt, etc.).

\section{Exclusion criteria}

1. Contraindications to MRI
(a) Pacemakers
(b) Intra-cranial aneurysm clips
(c) Claustrophobia

2. Causes of neck pain other than spondylosis
(a) Tumors
(b) Trauma
(c) Meningitis

\section{MRI imaging technique Acquisition}

1. Technique was performed using a standard 1.5-T MR unit (Intera and Achieva, Philips). A standard cervical coil was used. The sequences obtained were axial T1 and T2 WI, sagittal T1 and T2 WI, and diffusion tensor imaging.

2. DTI high isoSENSE sequence had the following parameters:
(a) A single shot, spin-echo echoplanar sequence in 33 encoding directions
(b) A diffusion weighting factor of 0 and $800 \mathrm{~s} / \mathrm{mm}^{2}$
(c) TR $2800 \mathrm{~ms}$
(d) TE $97 \mathrm{~ms}$
(e) Flip $90^{\circ}$

\section{Myelopathy detected by MRI \& DTI}

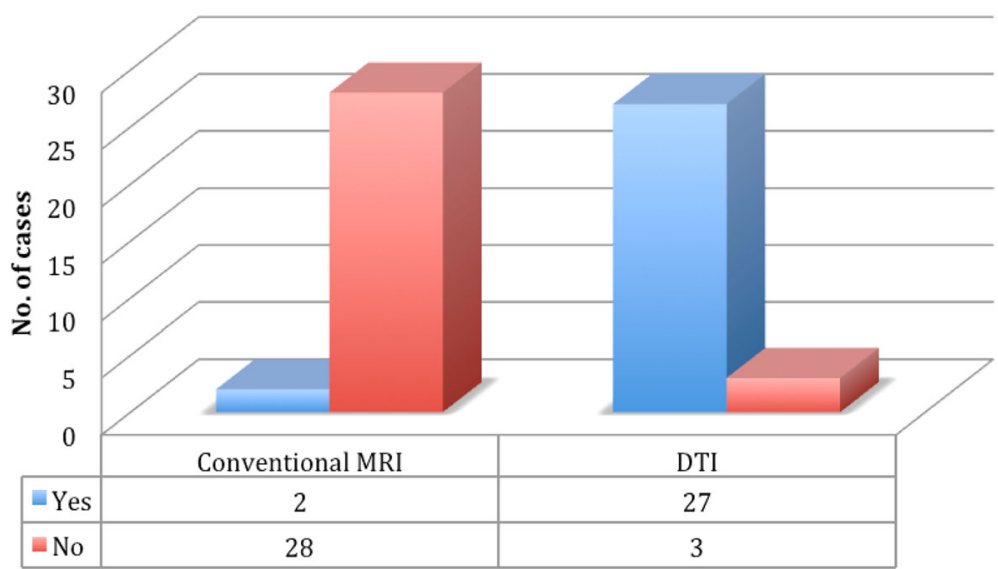

Fig. 1 Distribution of cases according to myelopathy detected with conventional MRI and DTI. DTI found to be $93 \%$ more sensitive in detecting early myelopathic changes than the conventional MRI 
Table 2 Comparison between mean FA values classified according to normal cord, cord opposite to most affected disc and cord below the most affected disc levels at different sites

\begin{tabular}{llll}
\hline & Normal & Affected disc & Below affected disc \\
\hline Whole cord section & 0.742 & 0.563 & 0.637 \\
3 o'clock & 0.802 & 0.601 & 0.685 \\
6 o'clock & 0.805 & 0.637 & 0.666 \\
9 o'clock & 0.823 & 0.624 & 0.662 \\
12 o'clock & 0.770 & 0.506 & 0.590 \\
\hline
\end{tabular}

(f) Matrix $80 \times 108$

(g) FOV $248 \mathrm{~mm}$

(h) Slice thickness: 2.0/00

(i) Acquisition plane: sagittal

All the images were transferred to the workstation (Philips extended MR workspace) supplied by the manufacturer.

\section{Processing}

- Radiological evaluation of the cervical spine and cord in axial and sagittal T1WI and T2WI images was done for detection of compressive myelopathy or cord caliber reduction.

- Then, DTI images were processed using the Philips software for tractography to obtain the color-coded FA maps in axial and sagittal planes.

- Assessment of the color-coded FA maps was done to detect any abnormality in the form of change in the normal blue code of the normally oriented craniocaudal cervical cord fibers.

- Multiple ROIs were manually drawn within the cervical cord in the axial combined anatomical and color-coded FA images opposite to all cervical discs levels.

- The drawn ROIs included only the cervical cord and we tried to exclude any surrounding CSF.

- FA values of all ROIs opposite to cervical discs were obtained.

- FA values at 3, 6, 9, and 12 o'clock positions of the spinal cord section were obtained at a normal cord segment (C2-C2/3), the most affected disc level and just below the level of the most affected disc.

- MR tractography was performed (multi-ROI technique), and the software algorithm tracked the white matter tracts that passed through these ROIs.

\section{Interpretation}

Two conjoint radiologists, with 5 and 10 years experience in neuroradiology, had interpreted the conventional and DTI MRI examinations of the cases with no discrepancy.

We used a simple grading system to quantify the effect of the discal lesion on the spinal cord:

- $0=$ disc not touching the cord.

- 1 = disc touching the cord.

- 2 = disc indenting the cord.

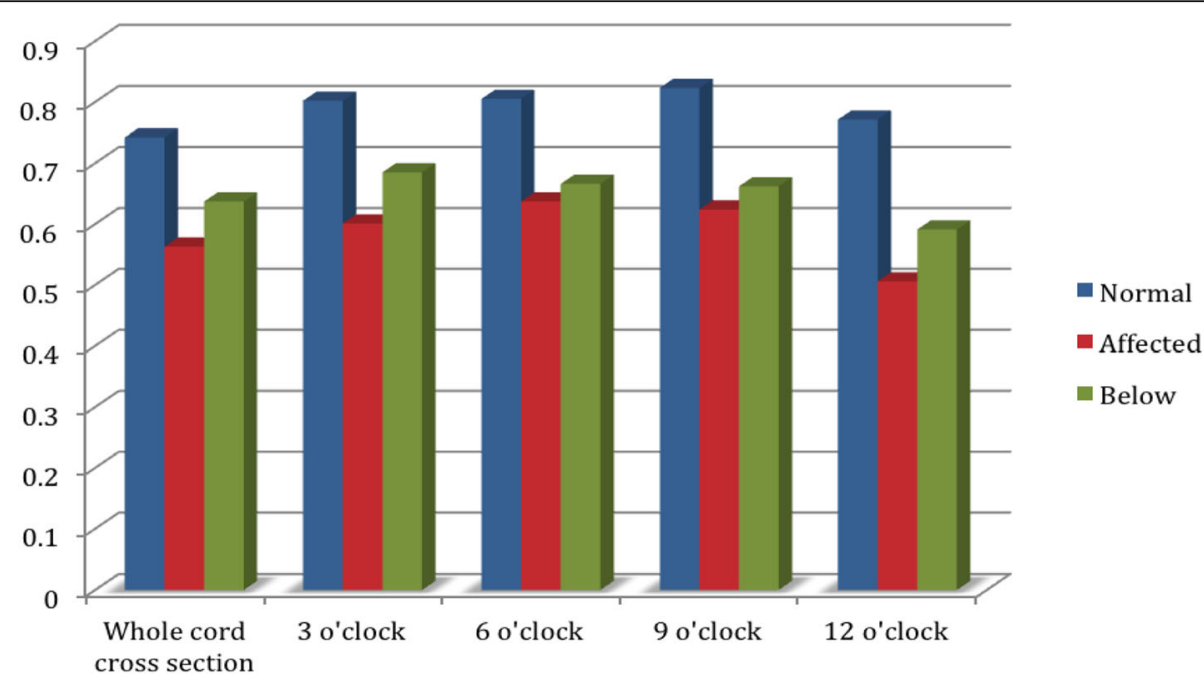

Fig. 2 Comparison between mean FA values classified according to normal cord, cord opposite most affected disc and cord below the most affected disc levels at different sites. The most significant reduction of FA value was found to be opposite the most affected disc level at 12 o'clock position 
The cervical cord myelopathy was diagnosed in conventional MRI by the presence of high T2 signal within the cord opposite to the disc lesion.

The cervical cord myelopathy was diagnosed in DTI MRI by the presence of change in the normal blue code of the normally oriented craniocaudal cervical cord fibers and/or by decreased FA value of the cervical cord opposite to the disc lesion compared to $\mathrm{C} 2$ level.

Score of the FA value reduction We used cutoff values to assess the severity of myelopathy based on FA values previously used in the study of Ellingson et al. [7] as follows:

- Mild: $<0.7$ to $\geq 0.5$

- Moderate: $<0.5$ to $\geq 0.3$

- Severe: $<0.3$

\section{Statistical analysis}

Data were statistically described in terms of mean standard deviation (SD), and range or frequencies (number of cases) and percentages when appropriate. Comparison between the different groups was done using paired $t$ test. For comparing categorical data, Chi square (2) and McNemar tests were performed. Exact test was used instead when the expected frequency is less than 5. $P$ values less than 0.05 were considered statistically significant. All statistical calculations were done using computer program SPSS (Statistical Package for the Social Science; SPSS Inc., Chicago, IL, USA).

\section{Results}

This study included 30 patients (11 males and 19 females); age ranged from 22 to 70 years (mean age $=44$ ).
The most frequent neurological clinical presentations given by the patients were neck pain and brachialgia (Table 1).

\section{Regarding the most affected disc}

C4-5 affection was seen in 14 cases (46\%), C3-4 affection was seen in 8 cases (27\%), C5-6 affection was seen in 5 cases (17\%), and C6-7 affection was seen in 3 cases $(10 \%)$.

\section{Regarding the cord deformity}

Thirteen cases showed no cord deformity (43.3\%), 11 cases showed discs touching the cord (36.7\%), and 6 cases showed discs indenting the cord (26.7\%).

\section{Regarding the detection of myelopathy (Fig. 1)}

- By conventional MRI: $2 / 30$ cases (6.7\%) showed myelopathy (Fig. 7), while 28/30 cases (93.3\%) showed no signal alteration of the cord.

- By DTI: $27 / 30$ cases (90\%) showed myelopathy by DTI, while $3 / 30$ cases (10\%) showed no myelopathy.

\section{Regarding the degree of myelopathy}

- The results obtained revealed that the mean FA value of the spinal cord opposite to the affected disc was the most decreased, and below the affected disc was decreased but less than opposite to the most affected disc (Table 2 and Fig. 2).

- The results also showed that the least FA value was opposite to 12 o'clock of the most affected disc (Fig. 6).

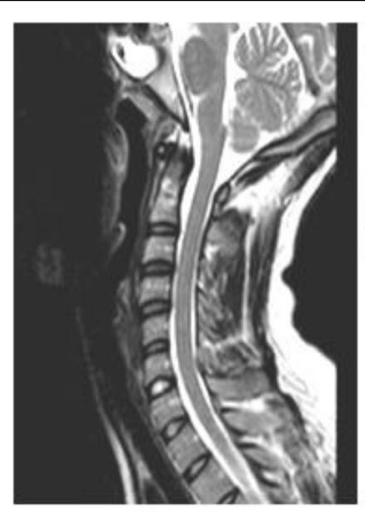

A

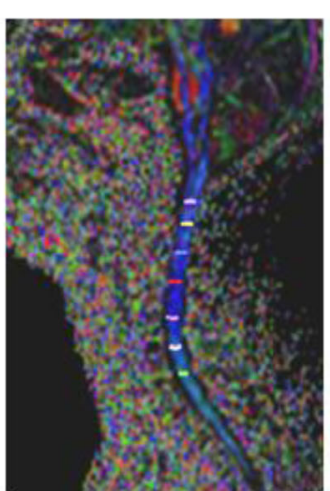

B

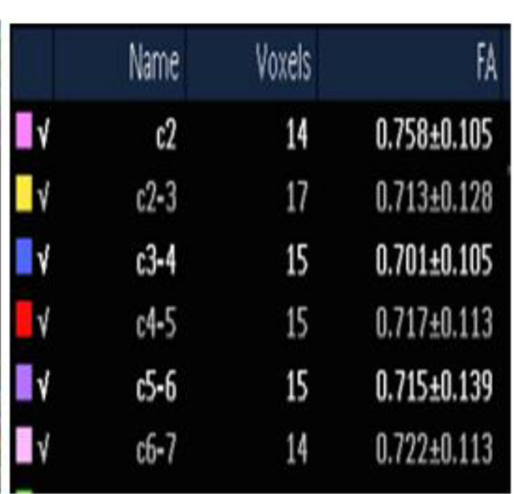

C

Fig. 3 Twenty-two-year-old female patient, presented with neck pain and bilateral brachialgia. a Sagittal T2 of the cervical spine showing no significant disc lesions. b Color-coded FA map of the cervical spinal cord showing no change in blue color with multiple ROls opposite to different disc levels. c FA values opposite to all the examined cervical discs with no gross abnormality 
- Comparing the FA values of the whole cord section, at 12, 3, 6, and 9 o'clock opposite to the most affected disc with the normal FA values, we found highly significant FA value reduction with $P$ value $=0.001$ (Figs. 3, 4, 5, 6, and 7).

- Comparing the FA values of the whole cord section, at 12, 3, 6, and 9 o'clock below the most affected disc with the normal FA values, we found highly significant FA value reduction with $P$ value $=0.001$ (Fig. 5)

- Comparing the FA values of the whole cord section, at 12 and 3 o'clock opposite to the most affected disc with the cord level below the most affected disc, we found highly significant FA value reduction with $\mathrm{P}$ value $=0.001$.

- Comparing the FA values at 6 and 9 o'clock of the cord opposite to the most affected disc with the cord level below the most affected disc, we found insignificant difference with $P$ value $=0.083$ and 0.075 , respectively.

\section{Discussion}

In this study, we obtained microstructural parameter (fractional anisotropy (FA)) of the cervical spinal cord in patients with suspected cervical compressive myelopathy (CSM) and compared DTI parameters (FA

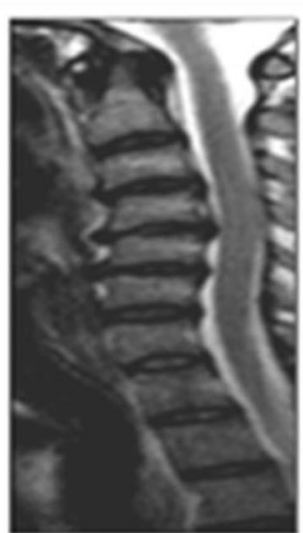

A

\begin{tabular}{|c|c|c|c|}
\hline If & a) & u & tmass \\
\hline I & or & u & tritum \\
\hline II & ats & s & I5stats \\
\hline 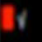 & 64 & $\mathrm{x}$ & thisar \\
\hline It & (b) & 5 & tomasu \\
\hline
\end{tabular}

D

\begin{tabular}{|rrrr|}
\hline$\vee$ & 12 & 1 & $0.388 \pm 0.000$ \\
$\vee \vee$ & 3 & 1 & $0.567 \pm 0.000$ \\
$\nabla \vee$ & 6 & 1 & $0.767 \pm 0.000$ \\
$\nabla \vee$ & 9 & 1 & $0.697 \pm 0.000$
\end{tabular}

$\mathbf{F}$

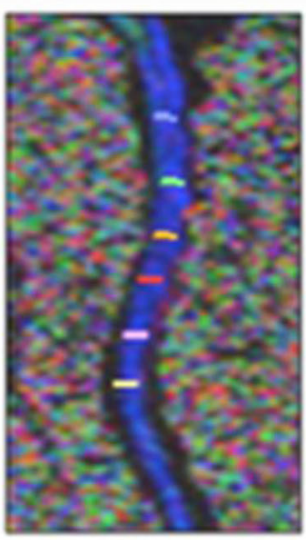

B

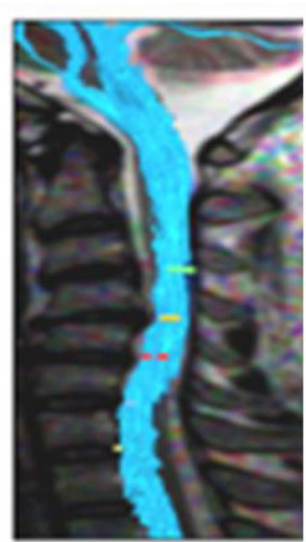

c

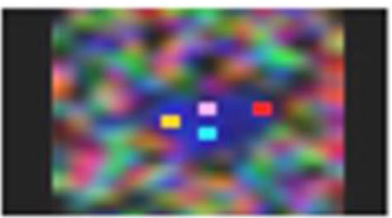

E

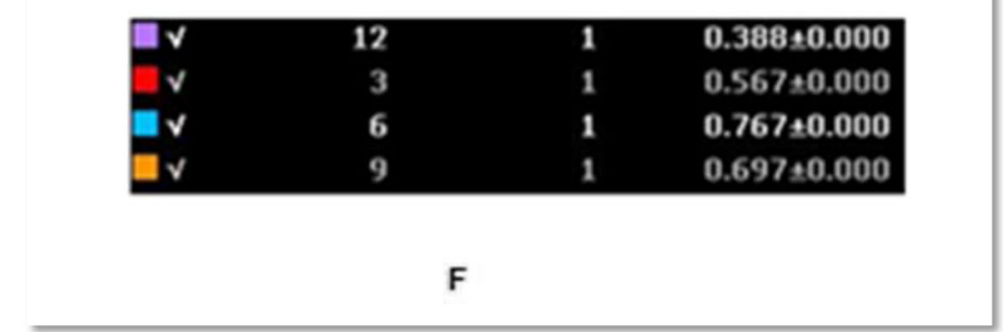

Fig. 4 Fifty-year-old female patient presented with neck pain. a Sagittal T2 WI of the cervical spine showing C3-4, C4-5, C5-6, and C6-7 posterior disc lesions, more prominent at C4-5 level, where it is seen indenting the cord, with no signal intensity alteration of the cord. $\mathbf{b}$ Color-coded FA map of the cervical cord with multiple ROls opposite to different disc levels. c DTI fiber tractography of the cervical cord showing grossly intact white matter fibers. d FA values opposite to all the examined cervical discs, showing mild reduction of FA value of the whole cord circumference opposite to the C4-5 disc level (the most affected disc level) denoting myelopathy. e, f FA values of the spinal cord at 3, 6, 9, and 12 o'clock positions opposite to C4-5 disc, showing the reduction in FA value is more pronounced opposite to 12 o'clock position 


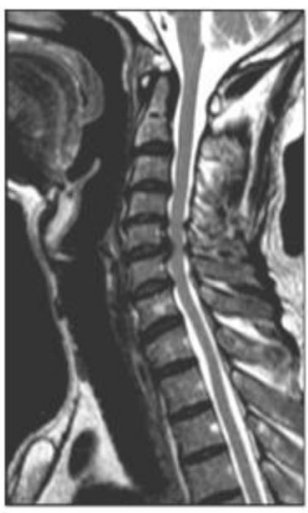

A

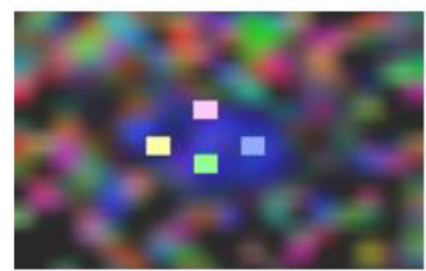

D

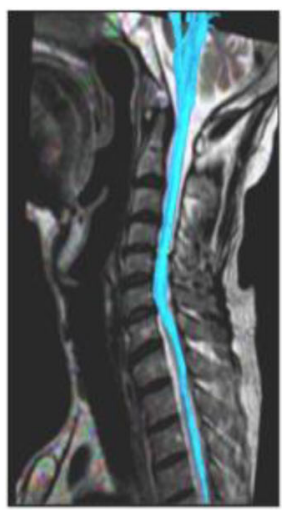

B

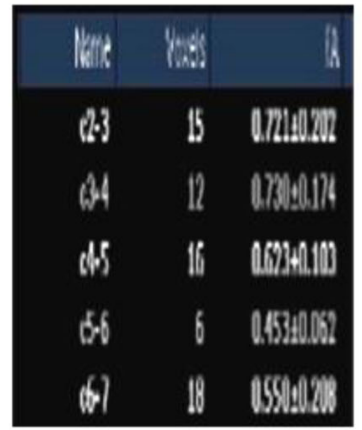

C

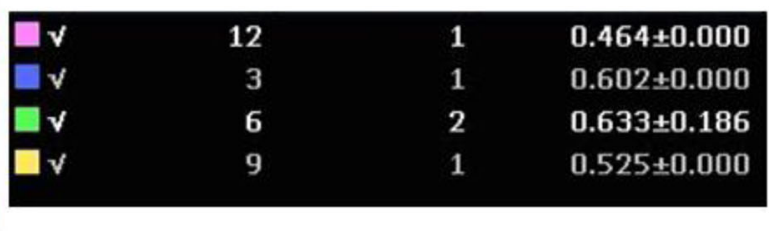

E

Fig. 5 Fifty-nine-year-old male patient, presented with neck pain and right brachialgia. a Sagittal T2 WI of the cervical spine showing C2-3 down to C6-7 posterior disc lesions with cord indentation at C5-6 level, with no signal intensity alteration of the cord. Ligamenta flava hypertrophy are seen at C4-5, C5-6, and C6-7 levels. b DTI fiber tractography of the cervical cord, showing grossly intact fiber tracts. c FA values opposite to all the examined cervical discs, showing moderate reduction of FA value of the whole cord circumference opposite to C5-6 level (the most affected disc level) denoting myelopathy and mild reduction opposite to C6-7 disc level (the level below the most affected disc). Mild reduction of FA value is seen opposite to C4-5 level as well. d, e FA values of the spinal cord at 3, 6, 9, and 12 o'clock positions opposite to C5-6 disc level, showing the reduction in FA value is more pronounced opposite to 12 o'clock

values) with the information obtained from conventional MRI sequences.

Our vision was to correlate DTI parameters (FA values) measured in patients' cervical cord levels opposite to normal and affected discs, as well as to identify the best location to measure FA values within the cord that reflects the severity of myelopathy.

Our study showed that DTI is $93 \%$ more sensitive in detecting early myelopathic changes than the conventional MRI. These results are consistent with Facon et al. [2], Kara et al. [4], Yoo et al. [6], Banaszek et al. [8] and Nukala et al. [9].

The mean FA value of the spinal cord opposite to normal disc levels in our study was 0.742 , compared to 0.745 as found by Facon et al, [2] and 0.734 by Uda et al, [10]. On the other hand, other studies showed variability in the mean FA values of the spinal cord ranging from 0.65 by Kara et al. [4] to 0.58 by Banaszek et al. [8].

In Facon et al.'s [2] study, the normal FA measurements were made in healthy volunteers at three different levels (cervical, C2-C5; high thoracic, T1-T6; and low thoracic, T7-T12) by using regions of interest located on the spinal cord. Special attention was paid to avoid CSF partial volume effect [2]. In our study, due to relative stability of the upper cervical region and lack of related discal lesions, we obtained a normal FA value for each patient at $\mathrm{C} 2-\mathrm{C} 2 / 3$ level, to use as internal reference to reduce the variability of FA values due to different ages and sex. Besides, we measured FA values opposite to all cervical disc levels not specific discs levels; we also paid special attention to avoid CSF partial volume effect.

In our study, we compared the mean FA values of the whole cord cross section opposite to normal cord level (C2) and most affected disc level. Our results showed highly significant reduction of FA values of the whole cord cross section, opposite to the level of the most affected disc $(P$ value $=0.001)$.

This matches with Song et al. [1], Hori et al. [3], and Kerkovský et al. [5] who reported significant FA reduction between compressed and non-compressed cord.

The anterior part of the spinal cord is the most affected site by degenerative myelopathy changes detected on DTI [11]. 


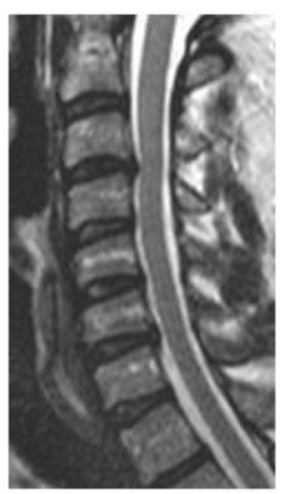

A

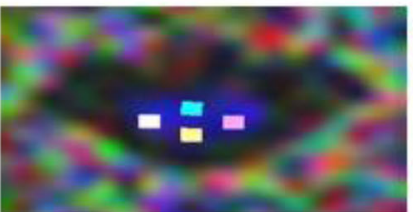

D

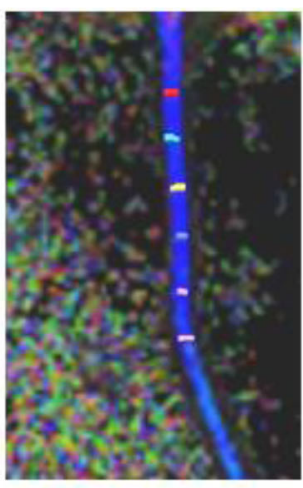

B

\begin{tabular}{|c|c|c|c|}
\hline IV & 2.3 & 10 & $0.782: 0239$ \\
\hline IV & $3-4$ & 10 & 0.72940 .179 \\
\hline IV & 0.5 & 11 & $0.779 \pm 0.206$ \\
\hline IV & 5.6 & 12 & 0.75240 .123 \\
\hline 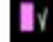 & 6.7 & II & $0.705+0.186$ \\
\hline
\end{tabular}

C

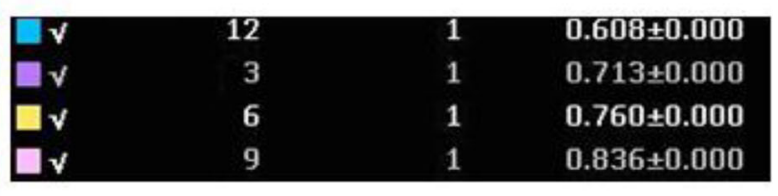

E

Fig. 6 Thirty-six-year-old male patient, presented with neck pain and bilateral brachialgia. a Sagittal T2 WI of the cervical spine showing C3-4 and C6-7 posterior disc lesions, just touching the ventral aspect of the cord at C3-4 level, with no signal intensity alteration of the cord. $\mathbf{b}$ Color-coded FA map, showing no significant changes of the blue color of the cord, with multiple ROls opposite to different disc levels. c FA values of the whole cord circumference opposite to all the examined cervical discs, showing no gross abnormality. d, e FA values of the spinal cord at 3, 6, 9, and 12 o'clock positions opposite to C3-4 disc, showing mild reduction in FA value opposite to 12 o'clock, denoting early myelopathy, although the normal FA value of the whole cord circumference

The anterior portion of the cord white matter opposite the affected disc level is more vulnerable to compressive myelopathic changes because it lies just posterior to the compressing factors such as degenerated discs and ossification of a posterior longitudinal ligament. So, DTI parameters (FA and ADC) are more affected at this site. On the other hand, DTI parameters in the posterior white matter remained unaffected. Also, the lateral white matter opposite to the affected level shows no significant changes of FA values, as the inflammatory cells proliferation and gliosis may help in its protection or reversal of damage [6].

When the spinal cord is displaced posteriorly by the spondylotic bar, the dentate ligaments resist this displacement and their dural attachments act as a fixed point, and this tensile stress is transmitted to the lateral columns which are more subjected to CSM while the anterior columns and the posterior columns are relatively unstressed [12].

We compared the mean FA values opposite to normal cord level (C2) and most affected disc level at 3, 6,9 , and 12 o'clock and we found that the most significant reduction of FA values was consistently noted at 12 o'clock position $(P$ value $=0.001)$. These findings agree with Yoo et al. [6] and Sąsiadek et al.
[11], and disagree with the dentate theory suggested by Levine [12].

By comparing FA values of the whole cord section, at 12, 3, 6 and 9 o'clock below the most affected disc with FA values opposite to normal cord level (C2-C2/3), we found highly significant FA value reduction with $P$ value $=0.001$. This matches with Kamble et al. [13] who found that FA values are decreased below the site of injury, likely due to Wallerian degeneration.

One limitation of the applied MRI protocol in this study was the relatively long scan time compared to the conventional MRI examination protocol which might represent burden for patients suffering from neck pain. Also, the processing of the DTI images was time consuming as we calculated the FA values of the whole cord circumference and opposite 3,6, 9, and 12 o'clock positions at multiple cervical disc levels.

Another limitation of this study was the relatively low signal to noise ratio of DTI images obtained with the 1.5$\mathrm{T}$ machine used in the study. We think that performing the examination using 3-T machines may improve the signal to noise ratio with more accurate assessment of DTI parameters and also the scan time can be shortened. 

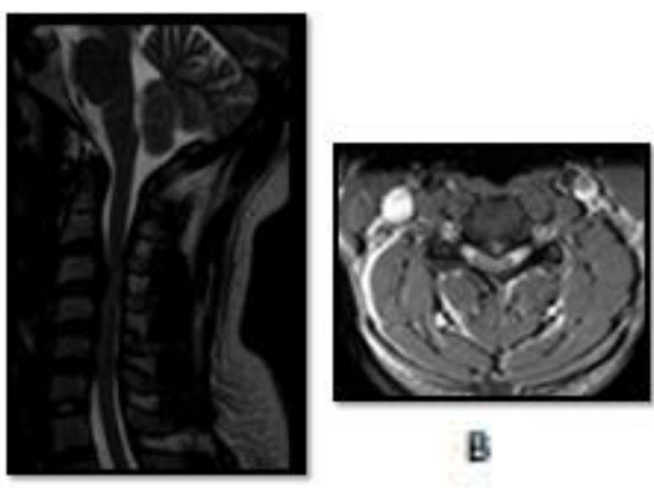

B
A

$\begin{array}{lll}0.3 & 15 & 0.714 \pm 0.173 \\ 0.4 & 19 & 0.436 \pm 0.105 \\ 0.5 & 18 & 0.355 \pm 0.933 \\ 6.6 & 18 & 0.552+0.138\end{array}$

D

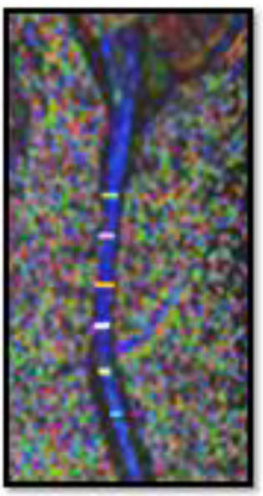

c

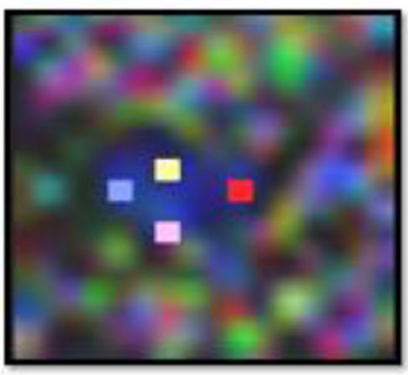

E

\begin{tabular}{|lrrr|}
\hline \hline$v$ & 12 & 1 & $0.412 \pm 0.000$ \\
\hline$v$ & 3 & 1 & $0.509 \pm 0.000$ \\
\hline$v v$ & 6 & 1 & $0.606 \pm 0.000$ \\
\hline$v$ & 9 & 1 & $0.618 \pm 0.000$ \\
\hline
\end{tabular}

$F$

Fig. 7 Thirty-three-year-old male patient, presented with paraesthesia in both upper limbs with motor weakness in right upper limb. a Sagittal T2 WI of the cervical spine and $\mathbf{b}$ axial T2 FFE opposite C3-4 level showing C4-5 and C5-6 posterior disc bulges indenting the cord and C3-4 posterior disc herniation with cranial migration with marked cord compression. The cervical cord is thinned and shows bright T2 signal opposite to C3-4 level denoting cord myelopathy. c Color-coded FA map, with multiple ROls opposite to different disc levels, showing change of the blue color of the cord opposite C3-4 level. d FA values of the whole cord circumference opposite to all the examined cervical discs, showing moderate reduction of FA value of the whole cord circumference opposite to C3-4 level (the most affected disc level) and C4-5 level (the level below the most affected disc) denoting myelopathy. Mild reduction of FA value is seen opposite to C5-6 level as well. e, f FA values of the spinal cord at 3, 6, 9, and 12 o'clock positions opposite to C3-4 disc level, showing the reduction in FA value is more pronounced opposite to 12 o'clock

\section{Conclusion}

Our study showed that DTI of the cervical spinal cord with FA measurement in patients with cervical spondylosis helps in early detection of cervical cord compressive myelopathy prior to appearance of changes in conventional MRI, which can help in treatment plans and improve the clinical outcome. Two-point FA measurements (the whole cord circumference and opposite to 12 o'clock) can be a simple tool for screening of early spondylotic cervical myelopathy.

\section{Abbreviations}

FA: Fractional anisotropy; CSM: Cervical spondylotic myelopathy; DTI: Diffusion tensor imaging; ADC: Apparent diffusion coefficient

\section{Acknowledgements}

Not applicable

\section{Authors' contributions}

All authors had actively contributed to the work. TAH contributed to the image revision, shared in the design and coordination of the study, revised the obtained results, and did drafting the manuscript. REA contributed to the idea and image revision, and revised the obtained results and data and final editing. SAB contributed to the 
data collection, obtained the radiological data in this study, and revised the obtained results. All authors read and approved the final manuscript.

\section{Funding}

No funding was received for this work from any organization.

\section{Availability of data and materials}

The datasets used and/or analyzed during the current study are available from the corresponding author on reasonable request.

\section{Ethics approval and consent to participate}

The study protocol was approved by the research committee of the radiology department, Faculty of Medicine, Cairo University, Egypt, on 13 October 2014 followed by the approval of the faculty committee in the same month (we do not have reference number, we have only the date of approval). An informed oral consent from each patient was taken before enrollment into the study.

\section{Consent for publication}

Written informed consent was obtained from all individuals (all were older than 16 years old) relevant to this research.

\section{Competing interests}

The authors declare that they have no competing interests.

\section{Author details}

${ }^{1}$ Radiology Department, Faculty of Medicine, Cairo University, Giza, Egypt. ${ }^{2}$ Radiology Department, Om El Masryeen Hospital (Public Health Institute), Giza, Egypt.

Received: 19 September 2019 Accepted: 29 October 2019

Published online: 28 November 2019

\section{References}

1. Song T, Chen WJ, Yang B et al (2011) Diffusion tensor imaging in the cervical spinal cord. Eur Spine J 3:422-428

2. Facon D, Ozanne A, Fillard P, Lepeintre JF, Tournoux-Facon C, Ducreux D (2005) MR diffusion tensor imaging and fiber tracking in spinal cord compression. AJNR 6:1587-1594

3. Hori M, Fukunaga I, Masutani Y et al (2012) New diffusion metrics for spondylotic myelopathy at an early clinical stage. Eur Radiol 8: 1797-1802

4. Kara B, Celik A, Karadereler S et al (2011) The role of DTI in early detection of cervical spondylotic myelopathy: a preliminary study with 3-T MRI. Neuroradiology 8:609-616

5. Kerkovský M, Bednarík J, Dušek L et al (2012) Magnetic resonance diffusion tensor imaging in patients with cervical spondylotic spinal cord compression: correlations between clinical and electrophysiological findings. Spine 1:48-56

6. Yoo WK, Kim TH, Hai DM et al (2013) Correlation of magnetic resonance diffusion tensor imaging and clinical findings of cervical myelopathy. Spine 8:867-876

7. Ellingson BM, Salamon N, Grinstead JW, Holly LT (2014) Diffusion tensor imaging predicts functional impairment in mild-to-moderate cervical spondylotic myelopathy. Spine 11:2589-2597

8. Banaszek A, Bladowska J, Szewczyk P, Podgórski P, Sąsiadek M (2014) Usefulness of diffusion tensor MR imaging in the assessment of intramedullary changes of the cervical spinal cord in different stages of degenerative spine disease. Eur Spine 7:1523-1530

9. Nukala M, Abraham J, Khandige G, Shetty BK, Rao AP (2019) Efficacy of diffusion tensor imaging in identification of degenerative cervical spondylotic myelopathy. Eur Radiol 6:16-23

10. Uda T, Takami T, Tsuyuguchi N et al (2013) Assessment of cervical spondylotic myelopathy using diffusion tensor magnetic resonance imaging parameter at 3.0 tesla. Spine 5:407-514

11. Sąsiadek MJ, Szewczyk P, Bladowska J (2012) Application of diffusion tensor imaging (DTI) in pathological changes of the spinal cord. Med Sci Monit 6:73-79

12. Levine DN (1997) Pathogenesis of cervical spondylotic myelopathy. J Neurol Neurosurg Psychiatry 62:334-340
13. Kamble RB, Neelam KV, Arun LN, Shailesh VR (2011) Diffusion tensor imaging in spinal cord injury. Indian J Radiol Imaging 3:221-224

\section{Publisher's Note}

Springer Nature remains neutral with regard to jurisdictional claims in published maps and institutional affiliations.

\section{Submit your manuscript to a SpringerOpen ${ }^{\circ}$ journal and benefit from:}

- Convenient online submission

- Rigorous peer review

- Open access: articles freely available online

High visibility within the field

- Retaining the copyright to your article

Submit your next manuscript at $\boldsymbol{\nabla}$ springeropen.com 